Result(s)* The research resulted in 782 eligible citations from January 2010 to October 2020. After the exclusion, nine articles that met all the inclusion criteria were included, comprising data from 1663 patients who underwent Radical Hysterectomy for Cervical Cancer IB1, the incidence of relapse was $10.6 \%$. Total patients who underwent open radical hysterectomy were $809(48.6 \%)$ with 75 relapses, patients who underwent minimally invasive surgery were 854 (51.4\%) and 105 relapses. When we compered the pattern of relapse local, Distant and Both of each group (open surgery and minimally invasive surgery), we did not see statistically significant differences (OD 0.963; 95\% CI, 0.602- 1.541; p=0.898), (OD $0.788 ; 95 \%$ CI, 0.467- 1.330; $\mathrm{p}=0.542$ ) and (CI 0.683; 95\% CI, 0.331- 1.407; $\mathrm{p}=0.630$ ) respectively.

Conclusion* There are no differences in patterns of relapse across surgical approaches in patients with stage IB1 cervical cancer undergoing radical hysterectomy as primary treatment.

\section{STAGE MIGRATION IN PATIENTS WITH LYMPH NODE POSITIVE CERVICAL CANCER}

${ }^{1} \mathrm{~T}$ Fahmy*, ${ }^{2} \mathrm{M}$ Persic. ${ }^{1}$ Barts and The London School of Medicine and Dentistry, United Kingdom; ${ }^{2}$ Royal Derby Hospital, United Kingdom

\subsection{6/ijgc-2021-ESGO.32}

Introduction/Background* Cervical cancer is the most common gynaecological malignancy worldwide. Despite strides in disease prevention with HPV (human papillomavirus) vaccination, and early detection of pre-cancerous changes, cervical cancer is nonetheless associated with high mortality. Survival is strongly linked to initial FIGO (International Federation of Gynaecology and Obstetrics) disease stage at diagnosis. In 2018, the FIGO staging criteria for cervical cancer were revised to include lymph node status, with positive nodes upstaging patients to stage $3 \mathrm{C}$ disease. This resulted in a retrospective stage migration for many patients. This study aims to analyse the effect of stage migration in cervical cancer on disease survival and systemic recurrence.

Methodology Data from a cohort of 76 cervical cancer patients from the University Hospital of Derby and Burton NHS Trust diagnosed and treated with chemoradiation and brachytherapy from 2012-2017 were collected. Patients with positive lymph nodes at diagnosis were assigned a new stage based on the current 2018 FIGO criteria and subsequently compared to patients whose stage at initial diagnosis remained unchanged.

Result(s)* $46 \%$ of patients were assigned a new higher stage based on lymph node status at diagnosis as per 2018 FIGO staging. An approximate $2.5 \mathrm{x}$ increase in cancer-related mortality was seen amongst those who had stage migrated versus those who remained the same stage $(37.14 \%$ versus $14.63 \%$, $p=0.024)$. Furthermore, a non-significant difference was seen in rates of systemic recurrence between the two groups, with around twice as many of the lymph node positive patients recurring within the timeframe of the study $(40.00 \%$ compared with $19.51 \%, p=0.05$ ).

Conclusion* These results reinforce the importance in the inclusion of lymph node status within 2018 FIGO staging criteria owing to the significant effect upon mortality in those who had stage migrated. The poorer prognosis and survival in the stage migration group also highlights the need for aggressive intervention in those with positive lymph nodes.

\section{DIAGNOSTIC PERFORMANCE OF PET-TC IN LYMPHATIC STAGING VS SURGICAL STAGING IN PATIENTS WITH LOCALLY ADVANCED CERVIX CANCER}

AF Rave Ramirez*, D González García-Cano, O Arencibia Sanchez, M Laseca Modrego, A Martín Martínez, T Benitez Delgado. Complejo Hospitalario Universitario Insular Materno Infantil de Gran Canaria, Gynecologic Oncology, las palmas de gran canaria, Spain

\subsection{6/ijgc-2021-ESG0.33}

Introduction/Background* The most important prognostic factor in cervical cancer is lymph node involvement. Up to $24 \%$ of patients with a negative CT scan show histological lymph node disease.The gold standard is histopathological study,but surgery is not exempt from possible complications (even laparoscopic approach). We intend to know the sensitivity and specificity of PET/CT in paraortic lymph node staging in LACC with negative CT.

Methodology Retrospective study of patients with LACC who have undergone laparoscopic paraortic lymphadenectomy and previously had performed a PET/CT. The indication for paraortic surgical staging in patients with LACC is a negative CT scan for disease at the paraortic level.

Result(s)* The mean age of the patients with cervical cancer was 52.7 years (range 23-97 years). 69\% were in advanced stages at the time of diagnosis. It should be noted that $35.5 \%$ of patients diagnosed in advanced stages underwent paraortic surgical staging. We have performed 227 laparoscopic paraortic lymphadenectomies in patients with LACC, 14\% with metastatic involvement. In 52 patients we had a PET/CT prior to paraortic lymphadenectomy. In the group of patients with negative PET ( $\mathrm{n}=45)$, we found that surgical staging was negative in $95.5 \%(\mathrm{n}=43)$. Meanwhile in patients with positive PET at the paraortic level $(n=7)$, we found that in 4 cases it was confirmed lymph node involvement in histology and in 3 cases they were false positives (predictive value $57.2 \%$ positive)

Conclusion* The specificity of PET/CT for paraortic lymph node staging in LCCA with Negative CT scan is $93.4 \%$, and the sensitivity is $66.6 \%$ in our series. If this trend is confirmed, we could consider modifying our strategy in the indication for paraortic surgical staging: in case of advanced stages with negative CT and PET/CT for lymphatic involvement, do not perform paraortic lymphadenectomy (negative predictive value of 95.5\%) and in cases of negative CT and positive PET, consider performing lymphadenectomy paraortic (positive predictive value of $57.2 \%$ ).

\section{ADJUVANT THERAPY AFTER RADICAL HYSTERECTOMY FOR EARLY CERVICAL CANCER. ARE WE HARMING OUR PATIENTS?}

F Boria*, N Manzour, E Chacon, N Martin, D Vazquez, T Castellano, JÁ Mínguez, JL Alcazar, A Gonzalez-Martin, L Sánchez Lorenzo, J Espinos, M Cambeiro, LM Chiva. Clinica universidad de navarra, Gynecologic Oncology, Spain

\subsection{6/ijgc-2021-ESG0.34}

Introduction/Background* In addition to positive pelvic lymph nodes, parametrial extension, and positive surgical margins, a combination of intermediate risk factors (Sedlis criteria) have been identified as poor prognostic factors. ${ }^{1-7}$ In these patients, adjuvant therapy is recommended in most of the clinical guidelines. However, indications for adjuvant therapy 